\title{
Therapeutic trials in adult FSGS: lessons learned and the road forward
}

\author{
An S. De Vriese, Jack F. Wetzels (i), Richard J. Glassock, Sanjeev Sethi(D) \\ and Fernando C. Fervenza
}

Abstract | Focal segmental glomerulosclerosis (FSGS) is not a specific disease entity but a lesion that primarily targets the podocyte. In a broad sense, the causes of the lesion can be divided into those triggered by a presumed circulating permeability factor, those that occur secondary to a process that might originate outside the kidneys, those caused by a genetic mutation in a podocyte or glomerular basement membrane protein, and those that arise through an as yet unidentifiable process, seemingly unrelated to a circulating permeability factor. A careful attempt to correctly stratify patients with FSGS based on their clinical presentation and pathological findings on kidney biopsy is essential for sound treatment decisions in individual patients. However, it is also essential for the rational design of therapeutic trials in FSGS. Greater recognition of the pathophysiology underlying podocyte stress and damage in FSGS will increase the likelihood that the cause of an FSGS lesion is properly identified and enable stratification of patients in future interventional trials. Such efforts will facilitate the identification of effective therapeutic agents.

Focal segmental glomerulosclerosis (FSGS) is a histopathological 'pattern of injury' seen on light microscopy (LM) - not a specific disease entity. It is conventionally defined as the consolidation of a portion (segmental) of the glomerular capillary tuft that is attached to Bowman's capsule and in late stages contains an accumulation of extracellular matrix, involving a subset (focal) of glomeruli ${ }^{1,2}$. The principal abnormality on electron microscopy (EM) is a variable degree of podocyte foot process effacement (FPE) and gaps in the podocyte cover of the glomerular basement membrane (GBM). Immune deposits are not present, but trapping of IgM and complement component $\mathrm{C} 3$ within the segmental sclerotic areas can be observed on immunofluorescence microscopy. The cardinal clinical feature is a variable degree of proteinuria with or without the nephrotic syndrome.

The FSGS lesion has heterogeneous causes $^{3}$. The common initiation event is podocyte damage, which ultimately results in podocyte depletion, and hence FSGS is considered to be a podocytopathy. It can be subdivided into four general categories: presumed permeability factor-related FSGS (ppfFSGS), secondary, genetic and unclassifiable (also termed FSGS of unknown cause) form $\mathrm{s}^{4,5}$.

A careful attempt to correctly stratify patients with FSGS based on their clinical presentation and pathological findings on kidney biopsy is essential for sound treatment decisions in individual patients and for the rational design of therapeutic trials in FSGS. Unfortunately, a number of clinical trials have failed to meet this dictum, and have enrolled a mixed population of FSGS categories without proper stratification. As a result, little progress has been made in the development of effective therapies. This Perspectives article provides a rationale for the stratification of FSGS based on current understanding of the pathophysiology of podocyte stress and damage. We envisage that the stratification of patients according to these categories will increase the likelihood that the cause of a FSGS lesion is properly identified, and improve the rational design and success of therapeutic trials.

\section{FSGS categories}

Presumed permeability factor-related FSGS. The form of FSGS traditionally termed 'primary FSGS' is presumed to be caused by a circulating permeability factor (or factors) that trigger(s) sudden and generalized injury to podocytes ${ }^{5}$. Given the causative role of the presumed permeability factor, we hereafter refer to this form of FSGS as ppfFSGS. Notably, despite intensive efforts, a definitive causative factor has not been conclusively identified, although several candidate molecules have been proposed, including cardiotrophinlike cytokine factor 1 (CLCF-1), soluble urokinase-type plasminogen activator receptor (suPAR), anti-CD40 antibody, apolipoprotein A1 and a soluble form of calcium/calmodulin-serine protein kinase $(\mathrm{CASK})^{6,7}$. A number of in vitro assays have indirectly demonstrated the presence of a circulating permeability factor in plasma from patients with active ppfFSGS, although these assays have not been validated and their use is currently limited to the experimental setting ${ }^{8,9}$. This form of FSGS is most commonly treated with immunosuppressive drugs, including glucocorticoids and calcineurin inhibitors $(\mathrm{CNIs})^{10}$ or with plasmapheresis or immunoadsorption. Whether the effects of the immunosuppressant drugs on proteinuria are due to a systemic or a local effect on podocytes remains uncertain (see below). Data from observational trials suggest that about $40-70 \%$ of patients with ppfFSGS respond to glucocorticoids or CNIs ${ }^{11}$, but these studies may have included patients with secondary and genetic forms of FSGS. We estimate that at least $70 \%$ of correctly classified patients with ppfFSGS should respond to a sufficiently long course of immunosuppression. Renin-angiotensin system (RAS) blockade is generally ineffective in reducing proteinuria in patients with ppfFSGS ${ }^{12}$. The recurrence rate of ppfFSGS is $70-80 \%$ in initial transplants when genetic and secondary causes are excluded ${ }^{13}$. It has been hypothesized that minimal change disease and ppfFSGS are part of the same disease spectrum, where both are associated with circulating permeability factors, but ppfFSGS represents a more advanced and often more therapy-resistant phenotype 
and minimal change disease a less severe and usually glucocorticoid-sensitive phenotype $\mathrm{e}^{14}$.

Secondary FSGS. Secondary FSGS is an umbrella term for FSGS lesions caused by a diverse array of pathogenic events and can be subdivided in maladaptive FSGS, drug-induced FSGS, viral-induced FSGS and FSGS lesions superimposed on other glomerular diseases. Most patients traditionally classified as having maladaptive FSGS have a distinctive and specific causative factor (for example, unilateral renal dysplasia or agenesis, congenital nephropenia or low nephron endowment, morbid obesity, a surgical reduction of renal mass or reflux nephropathy), but many others do not ${ }^{15}$. At least some of those individuals for whom a specific causative factor has not been identified may have an unidentified genetic form of FSGS, for example, caused by APOL1 risk alleles. Maladaptive forms of secondary FSGS are characterized by relative podocytopenia, resulting from a reduction in the number of functioning nephrons or from a normal nephron population subjected to an abnormal haemodynamic stress. In conditions associated with enlarged glomeruli (such as obesityrelated glomerulopathy or congenital nephropenia), the ratio of functioning podocytes to glomerular tuft surface area is decreased. Treatment of maladaptive FSGS is aimed at reducing injurious glomerular capillary hypertension, typically with RAS inhibitors; glucocorticoids and other immunosuppressive drugs are ineffective. Weight loss and caloric restriction reduce proteinuria in patients with obesity-related FSGS.
Other forms of secondary FSGS result from the direct effects of toxins (for example, bisphosphonates, interferons and androgens) or viral insults (for example, HIV, HCV and SARS-CoV-2) on podocytes ${ }^{16}$. FSGS lesions might also be seen in other glomerular diseases (for example, sclerotic lesions in IgA nephropathy, membranous nephropathy, lupus nephritis and ANCA-associated vasculitis), but the clinical phenotype is usually dominated by the primary disease. Secondary FSGS does not recur after transplantation.

Genetic FSGS. Defects in vital podocyte and GBM proteins are increasingly recognized as causes of FSGS (TABLE 1). Genetic FSGS may ensue from mutations in the nuclear genes that encode podocyte proteins involved in slit diaphragm structure and function, actin cytoskeleton architecture and regulation, nuclear function and cellular metabolic pathways and adhesion to the $\mathrm{GBM}^{17}$. Even more commonly, mutations in the structural GBM glycoproteins of the collagen IV lineage are causes of FSGS ${ }^{18}$. Future studies will probably identify causative mutations in novel genes, such as those involved in mitochondrial function or maintenance of the endothelial glycocalyx ${ }^{19}$.

Genetic FSGS is common in infants and young children and has been reported in up to $60 \%$ of children with steroid-resistant nephrotic syndrome ${ }^{20}$. However, adults with FSGS lesions can also harbour pathogenic mutations ${ }^{21}$. Large gene panels or whole exon sequencing can detect potentially pathogenic mutations in as many as $30 \%$ of adults with FSGS lesions ${ }^{22}$. Proteinuria is variable but can be high. Most adult patients with persistent

\section{Table 1 | Genes implicated in FSGS}

\begin{tabular}{l} 
Function of the gene product \\
\hline Slit diaphragm proteins \\
\hline Actin binding \\
\hline Actin regulation \\
\hline Nuclear transcription factors \\
\hline Nuclear pore complex proteins \\
\hline Mitochondrial proteins \\
\hline KEOPS complex (tRNA \\
modification) \\
\hline Lysosomal proteins \\
\hline Adhesion proteins \\
\hline $\begin{array}{l}\text { Glomerular basement } \\
\text { membrane proteins }\end{array}$ \\
\hline Other
\end{tabular}

Other

\section{Gene}

NPHS1, NPHS2, CD2AP, CRB2, TRPC6, FAT1

PLCE1, ACTN4, MYO1E, MYH9, INF2, ANLN, AVIL

ARHGDIA, ARHGAP24, KANK1, KANK2, KANK4, MAGI2, DLC1, ITSN1, ITSN2, DAAM2

LMX1B, WT1, SMARCAL1, NXF5

NUP93, NUP85, NUP107, NUP133, NUP160, NUP205, XPO5

COQ2, COQ6, COQ8B (ADCK4), PDSS2, MTTL1

OSGEP, TP53RK, TPRKB, LAGE3

SCARB2

ITGA3, ITGB4, LAMB2

COL4A3, COL4A4, COL4A5, COL4A6, LAMA5

SGPL1, CUBN, PTPRO, WDR73, EMP2, DGKE, ALG1 moderate-to-severe proteinuria progress to kidney failure, at variable rates ${ }^{17}$. Many patients with genetic FSGS - including those with mutations in COL4 genes respond favourably to RAS inhibitors ${ }^{23}$. Genetic FSGS is typically resistant to immunosuppression. Interestingly, patients with mutations in EMP2 (the protein product of which regulates CAVEOLIN-1 levels $)^{24}$ or in proteins that interact with Rho-like small GTPase, a key regulator of the actin cytoskeleton ${ }^{25}$, respond at least partially to glucocorticoids, suggesting that glucocorticoids can exert direct effects on podocyte function. Similarly, some mutations may respond to CNIs, although complete remissions are rare ${ }^{26,27}$. Whether these therapeutic responses were the result of direct actions of CNI on the podocyte actin cytoskeleton, for example, through regulation of synaptopodin phosphorylation $^{28}$, or secondary to the haemodynamic effects of CNI, is unknown. It is tempting to speculate that the variable effectiveness of CNIs among the genetic forms of FSGS relates to differences in the underlying podocyte abnormality. The most favourable response was observed in patients with mutations in WT1 (REF. ${ }^{27}$ ), which encodes a transcription factor that is essential for stabilization of the podocyte actin cytoskeleton. By definition, genetic FSGS does not recur after kidney transplantation. Rare cases of 'recurrent' proteinuria have been described in patients with mutations in NPHS1 (encoding nephrin), owing to the development of anti-nephrin antibodies following kidney transplantation ${ }^{29}$.

A number of susceptibility genes confer an increased risk of FSGS that manifests only when additional genetic or environmental 'second hits' occur. The best known of these are the G1 and G2 gain-of-function polymorphisms in the APOL1 gene $^{30}$. The remarkably high allele frequency in patients of sub-Saharan African ancestry is explained by the protective effects of these polymorphisms against trypanosomiasis. The APOL1 G1 and G2 variants increase the risk of progressive kidney disease in all conditions associated with podocyte injury, including FSGS, HIV-associated nephropathy, focal global glomerulosclerosis (FGGS), severe lupus nephritis and sickle cell nephropathy ${ }^{30}$. The terms APOL1 nephropathy and APOL1 podocytopathy have been coined to describe the phenotypical expressions caused by APOL1 gain-of-function mutations ${ }^{5,30}$. Mitochondrial dysfunction can also underlie increased podocyte 
susceptibility to injury. Mitochondrial cytopathies can cause adolescence-onset FSGS $^{31}$. Moreover, in patients with estimated glomerular filtration rate (eGFR) $>60 \mathrm{ml} /$ $\mathrm{min} / 1.73 \mathrm{~m}^{2}$, low mitochondrial DNA copy number - indicative of mitochondrial dysfunction - was associated with a higher risk of developing chronic kidney disease and microalbuminuria ${ }^{32}$. Mitochondrial dysfunction may thus prove to be a susceptibility factor similar to the APOL 1 risk variants.

FSGS of undetermined cause. Despite extensive evaluation, a clear aetiology cannot be determined for many patients with an FSGS lesion. The clinical presentation and EM findings of these patients are often similar to those of patients with maladaptive FSGS. A study of patients with FSGS in Olmsted County, MN, USA, showed that the cause of the lesion remained unknown in $>60 \%$ of patients with features characteristic of secondary FSGS ${ }^{15}$. We propose that such lesions are classified as FSGS of undetermined cause (FSGSuc) ${ }^{10}$. The term 'idiopathic' FSGS for this or any other form of FSGS should be strictly avoided, as it gives rise to confusion with primary FSGS (which we now propose to term ppfFSGS).

\section{Podocyte pathobiology}

Podocytes are highly specialized and terminally differentiated cells that help to govern the permselectivity of the glomerular filtration barrier. Their foot processes interdigitate with those of neighbouring cells and connect with specialized gap junctions that form the slit diaphragm. Any disturbance of this unique structure is consistently associated with loss of glomerular barrier function, leading to proteinuria. Podocytes are tethered to the underlying GBM by cell attachment molecules, such as integrins, but their continuous exposure to primary filtrate flow makes them vulnerable to detachment and loss in the urine. Podocytes are unable to proliferate and compensate for lost cells, and although it was once assumed that neighbouring podocytes could hypertrophy and/or migrate to cover denuded areas of the GBM, there is currently no evidence that gaps in the podocyte cover of the GBM are ever repaired ${ }^{33}$. Rather, it seems that neighbouring podocytes, and subsequently their neighbours as well, will be affected by podocyte loss, and ultimately detach, until the entire capillary loop is abandoned in a scarring process, resulting in the FSGS lesion ${ }^{33}$. Activated parietal epithelial cells may proliferate

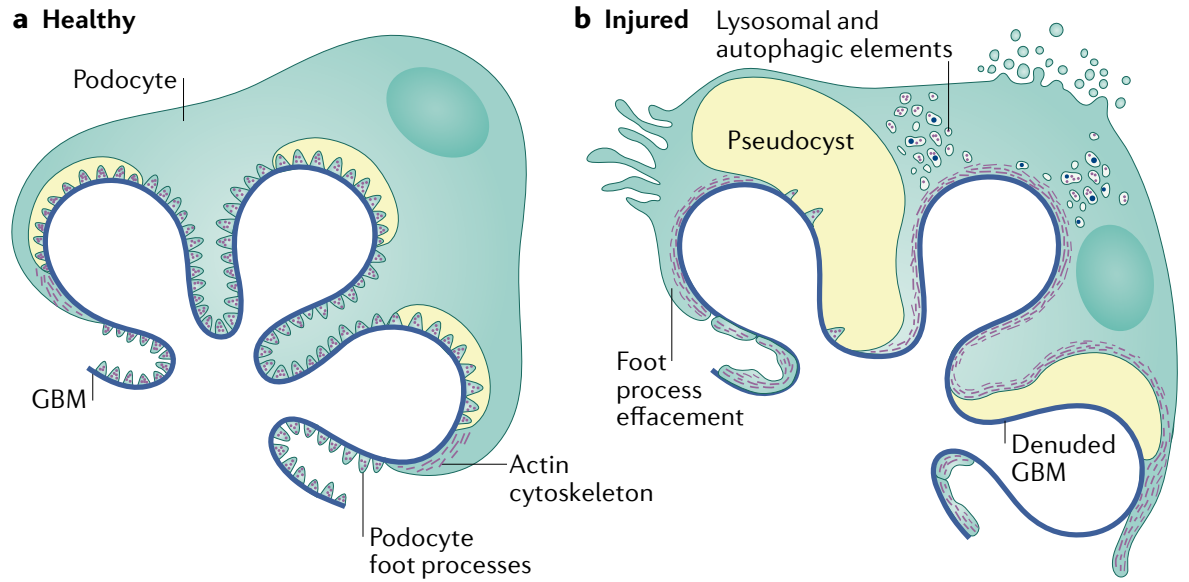

Fig. 1 | Podocyte structural changes in FSGS. a | Healthy podocytes are characterized by the presence of interdigitating foot processes and are attached to the underlying glomerular basement membrane (GBM) by attachment molecules. $\mathbf{b}$ | Characteristic structural changes in the injured podocyte include foot process effacement and detachment resulting in denuded areas of glomerular basement membrane. Image courtesy of Mayo Clinic.

and deposit extracellular matrix, thus also contributing to the formation of sclerotic lesions in FSGS ${ }^{34}$. Conversely, a subset of parietal epithelial cells may transdifferentiate towards podocytes and participate in a form of repair process with variable efficiency ${ }^{35,36}$. The balance between the regenerative and deleterious contributions of parietal epithelial cells might determine whether or not an FSGS lesion is formed.

Podocytes can be damaged through a variety of mechanisms, including toxin-mediated injury, viral infections, mechanical stress or through the effects of genetic mutations on podocyte or GBM function ${ }^{37}$. Podocytes respond to stress initially through loss of the interdigitating foot process pattern, termed FPE (FIG. 1) ) $^{38}$. This process represents a survival strategy to increase broad and firm adhesion of the podocyte to the underlying GBM and prevent detachment ${ }^{33}$. The elaborate podocyte actin cytoskeleton provides mechanical stability, enabling attachment of the podocyte to the GBM, but also allowing podocytes to rapidly change shape. FPE starts with closing of the filtration slits between neighbouring cells and proceeds with retraction, shortening and widening of the foot processes and attachment of the podocyte soma directly to the GBM, ultimately resulting in a continuous and flattened cytoplasmatic sheet that covers the GBM (FIG. 2) ${ }^{39}$. Our understanding of the way in which podocytes respond to different stressors provides a rationale to use information about the distribution and speed of FPE as a means of differentiating between subtypes of FSGS ${ }^{40}$.
Maladaptive FSGS occurs in conditions associated with hyperfiltration, glomerular capillary hypertension and glomerular hypertrophy. Although increased hydrostatic pressure was initially thought to be the main stressor of podocytes under these scenarios, we now understand that podocytes are particularly sensitive to shear stress ${ }^{41}$. In contradistinction to hydrostatic pressure, shear stress is unevenly distributed in the glomerular capillary, being highest in the initial segments and decreasing toward the end. This uneven distribution of shear stress explains why FPE is a slowly developing and heterogeneously distributed phenomenon in maladaptive FSGS ${ }^{15,42}$ (FIG. 3).

In ppfFSGS, on the other hand, a putative circulating permeability factor causes prompt generalized podocyte dysfunction and the resultant cytoskeletal dysregulation ensues in rapidly evolving and diffuse FPE (FIG. 3) ${ }^{15,42}$. In genetic FSGS, mutations in slit diaphragm or actin cytoskeleton proteins might directly induce dysfunctional changes in the cytoskeleton and cause diffuse FPE, whereas other mutations might render the podocyte more vulnerable to mechanical stress, resulting in segmental $\mathrm{FPE}^{17}$. In adults, mutations usually cause slowly developing podocyte injury and segmental FPE, whereas in children, defects in the slit diaphragm are generally more severe, resulting in diffuse FPE.

Although FPE and proteinuria are pathophysiologically linked, the precise mechanism of their relationship is undefined. No protein leakage can occur through the effaced cytoplasmic portions of the foot processes. However, gaps of unprotected GBM between the retracting 


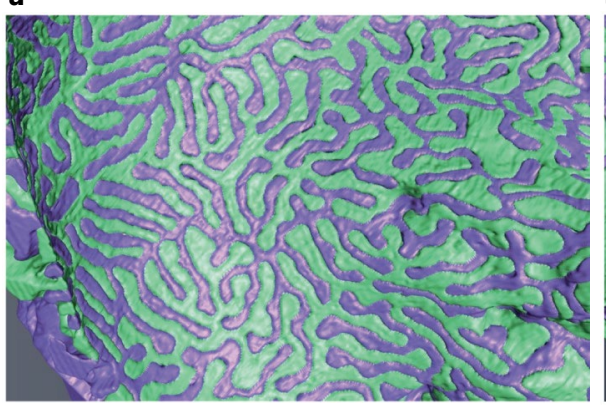

b

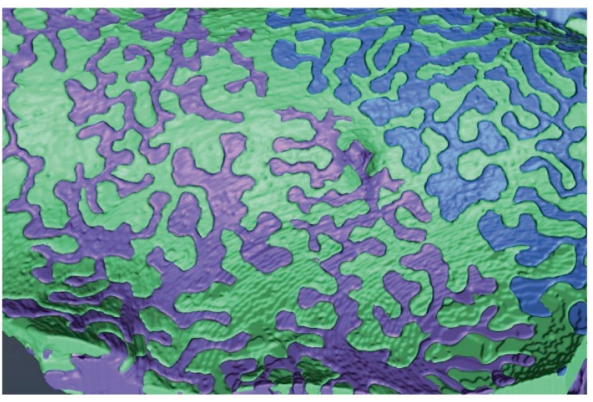

c

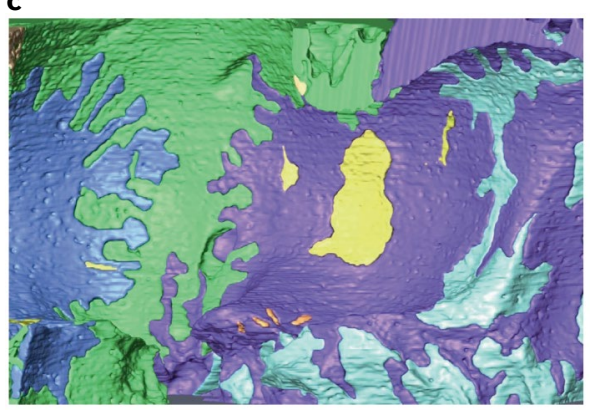

Fig. 2 | Foot process effacement in FSGS. Visualization of foot process effacement in podocytes using focused-ion beam/scanning electron microscopy tomography. Individual podocytes are shown in different colours. a | Foot processes of healthy rat podocytes exhibit a uniform width. $\mathbf{b} \mid$ In diseased podocytes (from rats with puromycin aminonucleoside-induced nephrosis), this uniformity in the foot processes width is lost. c | With disease progression, the podocytes form a large adhesive surface. The yellow masses represent cytoplasmic fragments without connection to neighbouring podocytes. Adapted with permission from REF. ${ }^{39}$, American Society of Nephrology.

foot processes may lead to non-selective and excessive filtration and proteinuria. The presence of proteinuria can therefore be interpreted as an undesirable consequence of the survival strategy of podocytes. In the later stages of the scarring process, however, FPE may reinforce the attachment of already damaged podocytes to the GBM and limit protein leakage across naked areas of GBM even if the defect will never be closed. This dual effect of FPE might explain why the degree of FPE and the level of proteinuria are only weakly correlated ${ }^{15}$. In contrast, a strong association exists between the degree of FPE and presence or absence of nephrotic syndrome. The overwhelming majority of patients with diffuse FPE have nephrotic syndrome, whereas nephrotic syndrome occurs only rarely if at all in patients with limited and heterogeneous FPE.

The widespread, toxic effects of the putative permeability factor(s) in patients with ppfFSGS are expected to affect practically every podocyte, resulting in generalized loss of the permselectivity barrier. This process is in line with our understanding of the clinical presentation of ppfFSGS, often characterized by sudden onset of severe nephrotic syndrome and oedema. On the other hand, patients with maladaptive FSGS may have nephrotic range proteinuria ( $>3.5 \mathrm{~g}$ per $24 \mathrm{~h}$ ), but serum albumin is usually normal and oedema is absent or develops gradually $y^{43,44}$. The cause of an FSGS lesion can be particularly difficult to determine in obese individuals ${ }^{45}$. In contradistinction to ppfFSGS, obesity-related FSGS is not associated with nephrotic syndrome even in the presence of massive proteinuria (>10-15g per day $)^{46}$. Thus, serum albumin concentration is essential for differentiating between ppfFSGS and maladaptive FSGS in these cases. Why patients with maladaptive FSGS do not develop hypoalbuminaemia remains unclear. One possibility is that the slow development of proteinuria in maladaptive FSGS enables the activation of compensatory mechanisms to counterbalance the loss of protein. Patients with genetic FSGS - particularly young children - can present with nephrotic syndrome. Clinical characteristics of adult patients with genetic FSGS are less well defined ${ }^{17}$, but adult patients can present with variable degrees of slowly developing proteinuria, and nephrotic syndrome is unusual ${ }^{18}$. In addition, adult patients with genetic FSGS often present with kidney function impairment and hypertension. Toxic and viral forms of secondary FSGS are usually associated with marked proteinuria, and nephrotic syndrome is also frequently present.

\section{Diagnostic evaluation of a FSGS lesion Light microscopy. The Columbia} classification categorizes FSGS lesions into five mutually exclusive types: collapsing, tip, cellular, perihilar and not otherwise specified $^{1}$. This classification is based on LM examination only and does not take into account the degree of FPE on EM. Although the Columbia classification has potential prognostic relevance, it should not be used as a tool to differentiate the different pathophysiological forms of FSGS. The not-otherwise-specified lesion, the most common subtype, can be present in all forms of FSGS. The collapsing subtype, associated with the worst prognosis, can occur in ppfFSGS, viral-induced or drugassociated FSGS. A tip lesion, generally found in therapy-responsive phenotypes and associated with the best prognosis, is thought to be the pathological corollary of heavy proteinuria and can appear in both ppfFSGS and maladaptive FSGS. Moreover, a specific lesion (for example, a perihilar lesion) associated with a certain pathophysiological state (such as hyperfiltration) can evolve over time into other lesions ${ }^{47}$. The collapsing and tip subtypes are generally associated with diffuse FPE on EM, but this association is not absolute. Taken together, LM alone cannot reliably distinguish ppfFSGS from the other forms of FSGS ${ }^{45}$, as epitomized by the presence of different lesions in a single biopsy (FIG. 4).

Electron microscopy. As described above, the (diffuse versus segmental) distribution of FPE is very useful in discriminating between the different forms of FSGS. EM is also a useful approach for ruling out other conditions that may erroneously be interpreted as a podocytopathy by LM alone (FIG. 5).

Of note, FPE cannot be reliably assessed by examination of glomeruli with advanced glomerulosclerosis. In addition, interpretation of FPE is rendered uncertain if the biopsy is taken during or after immunosuppressive treatment, particularly if partial remission has occurred. Also of note is the fact that even with EM it can be nearly impossible to determine if ppfFSGS has developed superimposed on other conditions associated with heavy proteinuria and FPE, such as diabetic nephropathy.

Although the importance of EM in the diagnostic work-up of an FSGS lesion cannot be overestimated, no standardized approach to the evaluation of FPE currently exists. A first glance at a low-power magnification $(\times 600-1,000)$ of $1-2$ glomeruli gives a good indication of the overall extent of FPE and should be followed by a closer look at a high magnification $(\times 1,800-5,000)$ for confirmation and evaluation of ultrastructural changes. Most pathologists perform a visual semi-quantitative estimation of the grade 
of FPE in the total available glomerular capillary surface in $5 \%$ or $10 \%$ increments (Vivette D’Agati, personal communication). Alternatively, pathologists may assess 10 capillary loops and estimate how many are affected by FPE (for example, $\geq 8$ of 10 loops with complete FPE is indicative of diffuse FPE). Exact measurements of foot process width across the total glomerular capillary surface has also been performed ${ }^{42,48}$.

However, as EM is infrequently used outside the renal pathology setting, a declining number of institutions are willing to invest in the required infrastructure. Alternative microscopic techniques to evaluate podocyte foot processes are therefore being developed. For example, in the past few years,

super-resolution imaging with 3D structured illumination microscopy (3D-SIM) has been used to visualize individual foot processes in human biopsy samples ${ }^{49,50}$. Quantification was achieved with software named Podocyte Exact Morphology Measurement Procedure ${ }^{50}$.

Proteinuria. The cardinal clinical feature of FSGS is proteinuria with or without nephrotic syndrome (traditionally defined as proteinuria $>3.5 \mathrm{~g}$ per day with serum albumin level $<35 \mathrm{~g} / \mathrm{l}(<3.5 \mathrm{~g} / \mathrm{dl})^{51}$, although some sources use $<30 \mathrm{~g} / \mathrm{l}(<3 \mathrm{~g} / \mathrm{dl})$ as the cut-off value for hypoalbuminaemia ${ }^{12,42,43}$. The lack of standardization of albumin assays complicates the classification of patients with FSGS and hinders between-study comparisons. For instance, bromocresol green assays markedly overestimate albumin level in patients with hypoalbuminaemia ${ }^{52}$. Pending standardization of albumin assays, we propose defining nephrotic syndrome as proteinuria $>3.5 \mathrm{~g}$ per day, with serum albumin $<35 \mathrm{~g} / \mathrm{l}(<3.5 \mathrm{~g} / \mathrm{dl})$ when measured using bromocresol green assay, and $<30 \mathrm{~g} / \mathrm{l}(<3.0 \mathrm{~g} / \mathrm{dl})$ when measured using bromocresol purple or immunonephelometric methods. If EM is unavailable, a reduced serum albumin level may be a surrogate marker for diffuse FPE.

\section{A clinicopathological approach}

A 'gold-standard' plasma or urinary biomarker that reliably identifies ppfFSGS and differentiates it from other causes of FSGS does not currently exist. The molecular nature of a putative circulating permeability factor(s) has also not yet been unequivocally identified ${ }^{5,53}$. Transcriptome and proteome profiling of whole glomeruli or single podocytes has been applied to unravel signalling pathways induced by podocyte stress ${ }^{54}$ and may enable the identification of patient subgroups that have a common underlying pathophysiology and respond to a specific therapeutic intervention ${ }^{55}$. However, the translation of these findings into clinical practice remains an area for future study.

At present, patient stratification through the identification of prototypical clinical and pathological characteristics of the different
FSGS subtypes is flawed. The absence of sound diagnostic criteria with which to identify patients in epidemiological and mechanistic studies and enrol patients in therapeutic trials inherently invalidates the conclusions from these studies. As a consequence, the development of a 'gold-standard' diagnostic test and targeted therapies is undermined. It could be argued that in the absence of specific biomarkers, a diagnosis of a specific FSGS lesion on kidney biopsy cannot be established. This view, although technically correct, has the potential to lead to nihilism in the approach to patients with FSGS. To interrupt a vicious circle of diagnostic and therapeutic uncertainty, we propose a number of axioms derived from insights into the pathophysiology of podocyte injury (TABLE 2). We suggest that these axioms, although unproven, can serve as a starting point for further study and reasoning. This approach has potential to move the field forward, pending the availability of 'gold-standard' biomarkers with which to stratify patients according to their underlying pathophysiology.

ppfFSGS is a clinico-pathological diagnosis, derived after a comprehensive evaluation of clinical characteristics, laboratory findings and careful review of the kidney biopsy sample, always including EM. We strongly submit that the diagnosis 'biopsy-proven primary FSGS' cannot be made using examination by LM alone.
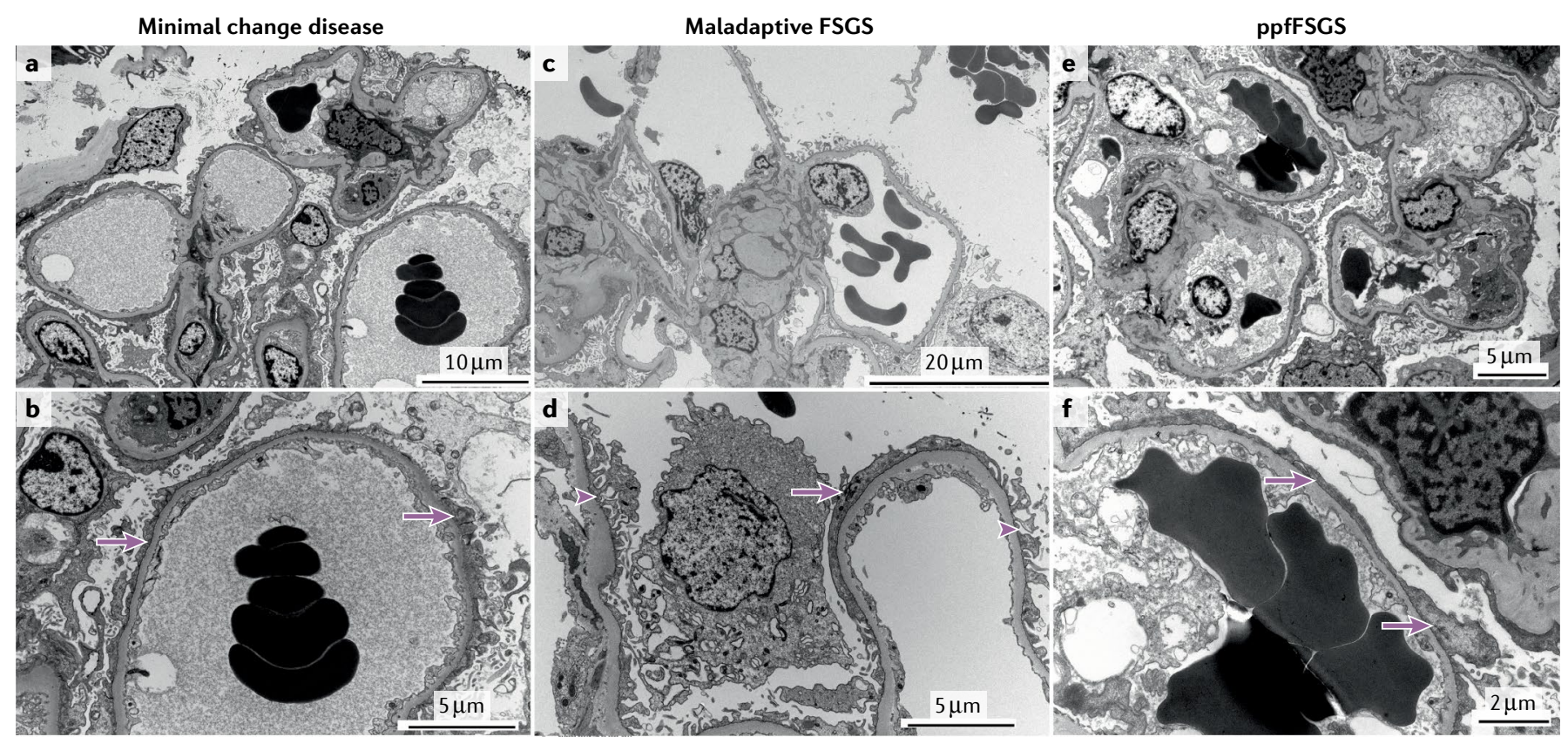

Fig. 3 | Ultrastructural visualization of foot process effacement. a,b | Minimal change disease showing diffuse foot process effacement (arrows). $\mathbf{c , d} \mid$ Maladaptive focal segmental glomerulosclerosis (FSGS) showing both preserved (arrowhead) and effaced (arrow) foot processes. e,f $\mid$ Presumed permeability factor FSGS (ppfFSGS) showing diffuse foot process effacement (arrows). 


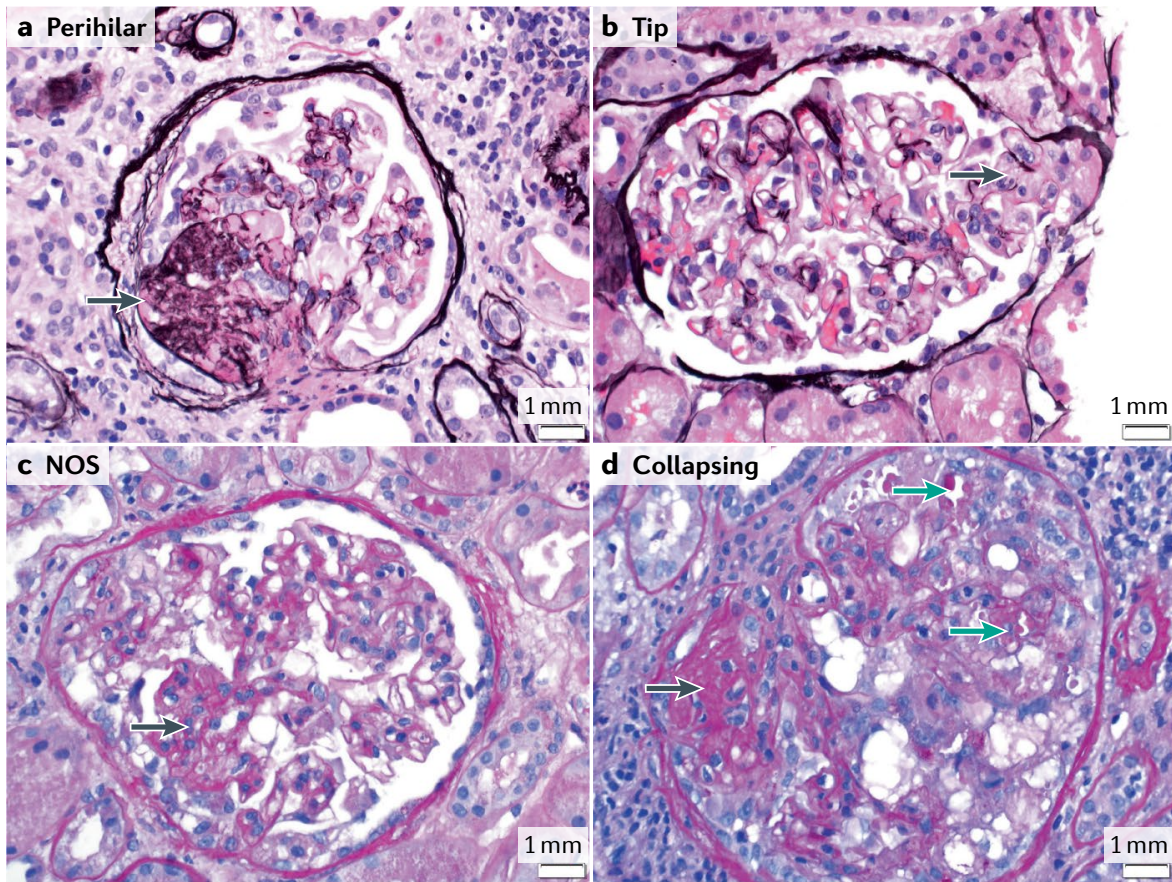

Fig. 4 | FSGS findings by light microscopy. A 41-year-old man with a history of sickle cell disease presented with shortness of breath, pleural effusions and lower extremity oedema. His weight was $120.7 \mathrm{~kg}$, serum creatinine $177 \mu \mathrm{mol} / \mathrm{l}(2 \mathrm{mg} / \mathrm{dl})$, serum albumin $34 \mathrm{~g} / \mathrm{l}(3.4 \mathrm{~g} / \mathrm{dl})$, proteinuria $12 \mathrm{~g} / 24 \mathrm{~h}$. Serology for hepatitis and HIV were negative. Light microscopy of kidney biopsy showed perihilar (a), tip (b), not otherwise specified (NOS) (c) and (d) collapsing lesions. a,b: silver methenamine stain, c,d: periodic acid Schiff stain; magnification $\times 40$. Black arrows show areas of segmental sclerosis; green arrows show a collapsing lesion with epithelial cell hypertrophy and protein reabsorption granules within the epithelial cells. The presence of different Columbia classification subtypes in a single biopsy underlines the inability of light microscopy alone to classify a focal segmental glomerulosclerosis lesion aetiologically. Electron microscopy showed diffuse foot process effacement. The diagnosis of presumed permeability factor focal segmental glomerulosclerosis was made.

All too often, a label of 'primary FSGS' has been erroneously given when identifiable causes of secondary FSGS were excluded and a genetic analysis yielded negative results. Maladaptive FSGS can be strongly suspected based on morphological findings. Toxic and viral forms of secondary FSGS can resemble ppfFSGS, but can be differentiated by a careful history and serological studies. Genetic FSGS must be suspected in all cases of FSGS that are unresponsive to steroids, in which biopsy findings reveal pathological alterations in GBM structure, or that involve a family history of proteinuria. FSGSuc accounts for a substantial but variable fraction of patients with an FSGS lesion, depending on the vigour of the evaluation, and is a diagnosis of exclusion.

\section{Categories of therapeutic agents}

The key to a successful intervention trial in FSGS lies in the recruitment of only those patients who are expected to respond to the agent under study. On this premise, we can apportion potential therapeutic agents into six main categories based on their cellular target. We discuss which FSGS subtypes may derive benefit from treatment with these agents and why some trials have yielded disappointing or misleading results. Of note, individual agents - such as sparsentan, steroids, CNIs, rituximab, adrenocorticotropic hormone (ACTH) and abatacept - may belong in more than one category.

A major drawback in the development of novel therapies for FSGS is a lack of animal models that are representative of the different FSGS subtypes. Thus, when a particular signalling pathway associated with podocyte stress is identified in an experimental setting, it remains unclear whether interference with this pathway will be non-selectively beneficial in all podocytopathies or only affect a specific subtype. This uncertainty reinforces the unassailable importance of proper patient stratification in clinical trials.

\section{Targeting the permeability factor(s).}

Treatments in this first category (category I) are developed to remove or inhibit the action of putative permeability factor(s) underlying ppfFSGS. By definition, they are expected to show benefit in ppfFSGS without any benefit in secondary or genetic FSGS.

This category includes extracorporeal treatment procedures that share the objective of removing the putative permeability factor(s). In patients with post-transplantation recurrence of FSGS, plasmapheresis $s^{56}$ and immunoadsorption ${ }^{57}$ have achieved high remission rates and are currently part of the standard treatment regimen. Selective LDL apheresis, although primarily intended to rapidly correct dyslipidaemia in patients with nephrotic syndrome, has been shown to reduce proteinuria in patients with FSGS an effect that was attributed to removal of the putative permeability factor $(\mathrm{s})^{58}$. The technique has been applied mainly in therapy-resistant FSGS, yielding mixed results ${ }^{58}$. Similarly, unselective immunoadsorption was successful in only a minority of patients with refractory FSGS ${ }^{58}$. The inconsistent outcome of extracorporeal treatment procedures in native FSGS can probably be attributed to the inclusion of unrecognized genetic forms of FSGS, which are highly prevalent among patients with therapy-resistant disease or due to the initiation of treatment in late-stage disease, when podocyte depletion has reached a point of no return.

It has been hypothesized that the putative permeability factor(s) may have a lectin-like interaction with sugars of the podocyte glycocalyx leading to signal transduction in podocytes. Free galactose has been reported to bind and inactivate the putative permeability factor(s) $)^{59}$. In one randomized controlled trial (RCT) of galactose versus standard medical therapy in patients with 'biopsy-confirmed primary FSGS', only 2 of 7 patients treated with galactose reached the primary end point of a $50 \%$ reduction in proteinuria - a proportion that was identical to that achieved with standard therapy ${ }^{60}$. Our review of the patient characteristics suggests that several patients did not have nephrotic syndrome at baseline and therefore may not have had ppfFSGS.

CD40 antagonism might also have a role in the treatment of ppfFSGS. CD40 is a co-stimulatory protein that is expressed by antigen-presenting cells and is also constitutively expressed by podocytes. Blocking interaction of CD40 with its ligand CD40L has protective effects in animal models of FSGS ${ }^{61}$. Serum of patients with recurrent FSGS post-transplantation contains anti-CD40 antibodies that are activating and pathogenic in vitro and 
in vivo, whereas a commercial anti-CD40 antibody inhibits this effect ${ }^{62}$. A phase 2 trial is currently underway to investigate the efficacy of the anti-CD40 monoclonal antibody bleselumab in preventing post-transplantation recurrence of FSGS (NCT02921789).

\section{Suppressing permeability factor formation.}

A second category of agents (category II) are those intended to interfere with the innate or adaptive immune response and suppress formation of the putative permeability factor(s). Agents in this category would be expected to be effective in the treatment of ppfFSGS only, without benefit in secondary or genetic FSGS.

Glucocorticoids are the current cornerstone of treatment in ppfFSGS ${ }^{10}$ and indeed, glucocorticoid responsiveness identifies an FSGS lesion as ppfFSGS. CNIs are standard therapy for patients with contraindications or intolerance to glucocorticoids and in patients who are glucocorticoid-resistant ${ }^{10}$. The mechanism of action of glucocorticoids and CNIs in ppfFSGS is not well understood, but it is presumed that these agents interfere with the cellular sites at which the putative permeability factors are produced. Direct effects on podocytes have also been suggested. Although glucocorticoid sensitivity is fairly specific for ppfFSGS, it is unfortunately not a very sensitive marker of ppfFSGS. The discrimination of glucocorticoid-resistant ppfFSGS from secondary FSGS, genetic FSGS and FSGSuc, which by definition are glucocorticoid-resistant, is challenging. Glucocorticoid resistance is a common but unfortunately variably defined, entry criterion for clinical trials of potential ppfFSGS therapies. For example, a RCT conducted in 138 patients aged $2-40$ years with glucocorticoid-resistant FSGS did not find a difference in remission rates between a 12-month course of cyclosporine $(n=72)$ or a combination of oral pulse dexamethasone and mycophenolate mofetil $(n=66)^{63}$. No EM evaluation of biopsy samples was performed. Rather, this trial and a descriptive follow-up study ${ }^{64}$ assessed treatment effects using LM evaluation of the FSGS lesion as a starting point. Moreover, levels of proteinuria and albuminemia at inclusion suggest that a substantial proportion of participants did not have nephrotic syndrome, and therefore were unlikely to have ppfFSGS. $70 \%$ of patients were younger than 18 years of age, $10 \%$ had a family history of kidney disease and $38 \%$ were African American, suggesting that many participants may have had unrecognized genetic FSGS or an APOL1 risk variant. In addition, glucocorticoid resistance was determined after only 4 weeks of prednisone therapy rather than the recommended 16 weeks, allowing inclusion of patients with ppfFSGS who had a slow response to steroids. Taken together, this study probably included a heterogeneous group of patients with different forms of FSGS, including ppfFSGS with partial remission to steroids, secondary FSGS and genetic FSGS. As such, true differences in treatment efficacy in patients with ppfFSGS may have been obscured.

Although not proven, the anti-CD20, $B$ cell-depleting antibody rituximab may reduce the elaboration of putative permeability factor(s) or have direct beneficial effects on podocytes. However, patients with steroid-resistant FSGS usually also fail to respond to treatment with rituximab ${ }^{65}$. Rituximab has not been studied as initial therapy for ppfFSGS, but it has beneficial effects in relapsing minimal change disease and in post-transplantation recurrent FSGS ${ }^{66,67}$.
Targeting haemodynamic abnormalities.

A third category of therapeutic agents (category III) are intended to correct the haemodynamic abnormalities that promote podocyte shear stress, and are expected to be primarily but not exclusively beneficial for maladaptive FSGS.

RAS inhibitors non-specifically attenuate proteinuria in all forms of FSGS (but least effectively in ppfFSGS), primarily by reducing the transglomerular hydraulic pressure gradient. Their effects are short lived and lasting remission requires continued administration. RAS inhibitors might also have direct effects on podocytes, as these cells possess a full complement of RAS components ${ }^{68}$.

Sparsentan is a dual endothelin type A (ETA) and angiotensin II type 1 receptor antagonist. Podocytes express both ETA and ETB receptors, and treatment with endothelin receptor antagonists prevents disruption of the actin cytoskeleton in experimental FSGS ${ }^{69}$. Thus, sparsentan may be of benefit in patients with FSGS beyond its blood pressure and intraglomerular pressure-lowering effects. The effects of
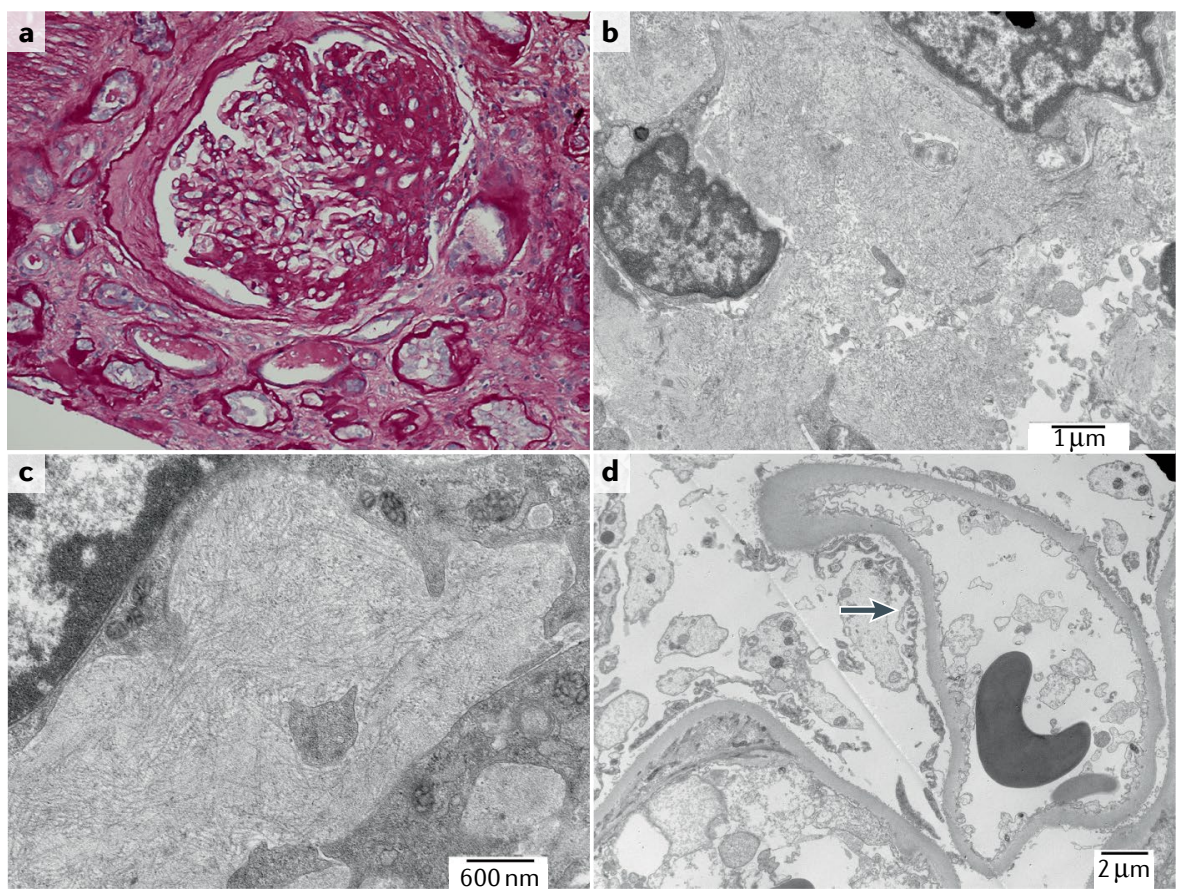

Fig. 5 | Electron microscopy evaluation of FSGS. A 47-year-old man presented with serum creatinine $186 \mu \mathrm{mol} / \mathrm{l}(2.1 \mathrm{mg} / \mathrm{dl})$, serum albumin $44 \mathrm{~g} / \mathrm{l}(4.4 \mathrm{~g} / \mathrm{dl})$ and proteinuria $3.4 \mathrm{~g} / 24 \mathrm{~h}$. a | Light microscopy showed perihilar segmental sclerosis. Periodic acid Schiff stain; magnification $\times 40$. b | Electron microscopy demonstrated randomly arranged fibrils $15 \mathrm{~nm}$ in diameter (that stained Congo red negative, not shown), consistent with a diagnosis of fibrillary glomerulonephritis; magnification $\times 11,000$. c| These fibrils were even more noticeable at higher magnification $(\times 23,000)$. d | Segmental foot process effacement (arrow); magnification $\times 6,800$. Electron microscopy enabled a diagnosis of fibrillary glomerulonephritis to be made and avoided an erroneous diagnosis of focal segmental glomerulosclerosis (FSGS) based on light microscopy alone. 
Table 2 | Axioms that define FSGS subtypes

\begin{tabular}{|c|c|c|c|c|}
\hline Axiom & $\begin{array}{l}\text { Presumed } \\
\text { permeability } \\
\text { factor-related } \\
\text { FSGS (ppfFSGS) }\end{array}$ & Maladaptive FSGS ${ }^{a}$ & Genetic FSGS & $\begin{array}{l}\text { FSGS of undetermined } \\
\text { cause }\end{array}$ \\
\hline $\begin{array}{l}\text { Findings on LM } \\
\text { (beyond the } \\
\text { FSGS lesion) }\end{array}$ & $\begin{array}{l}\text { Generally, no other } \\
\text { damage unless late } \\
\text { in disease course }\end{array}$ & $\begin{array}{l}\text { Often FGGS; varying degrees } \\
\text { of chronic damage, perihilar } \\
\text { lesions or glomerulomegaly } \\
\text { may be present but are not } \\
\text { diagnostic in themselves }\end{array}$ & Varying degrees of chronic damage & $\begin{array}{l}\text { Often FGGS; varying degrees } \\
\text { of chronic damage }\end{array}$ \\
\hline $\begin{array}{l}\text { Extent of } \\
\text { foot process } \\
\text { effacement } \\
\text { on EM }\end{array}$ & $\begin{array}{l}\text { Generalized }(>80 \%) \\
\text { in non-sclerotic } \\
\text { glomeruli }\end{array}$ & Mild and segmental & $\begin{array}{l}\text { Either segmental or diffuse. GBM } \\
\text { alterations may be prominent in type IV } \\
\text { collagenopathies }\end{array}$ & Mild and segmental \\
\hline $\begin{array}{l}\text { Response to } \\
\text { RAS inhibition } \\
\text { (or sparsentan) }\end{array}$ & Poor & Excellent & $\begin{array}{l}\text { May be good, but has not been } \\
\text { rigorously tested }\end{array}$ & Good \\
\hline $\begin{array}{l}\text { Glucocorticoids } \\
\text { and CNls }\end{array}$ & $\begin{array}{l}\text { May induce } \\
\text { remission }\end{array}$ & $\begin{array}{l}\text { Ineffective and potentially } \\
\text { harmful }\end{array}$ & $\begin{array}{l}\text { Ineffective. Response to CNIs is } \\
\text { anecdotal }\end{array}$ & Ineffective \\
\hline $\begin{array}{l}\text { Genetic tests } \\
\text { and family } \\
\text { history }\end{array}$ & Unrevealing & Unrevealing & $\begin{array}{l}\text { May reveal mutations in podocyte or } \\
\text { GBM proteins. Negative tests do not } \\
\text { exclude a genetic cause }\end{array}$ & Unrevealing \\
\hline $\begin{array}{l}\text { Underlying } \\
\text { cause }\end{array}$ & $\begin{array}{l}\text { No evidence of a } \\
\text { causative factor } \\
\text { (e.g. cancer, } \\
\text { auto-immunity, viral } \\
\text { infection, toxins) }\end{array}$ & $\begin{array}{l}\text { Evidence of a causative } \\
\text { factor or process (e.g. } \\
\text { unilateral renal dysplasia or } \\
\text { agenesis, sickle cell disease, } \\
\text { reflux nephropathy, obesity, } \\
\text { healing phase of proliferative } \\
\text { glomerulonephritis) is present }\end{array}$ & $\begin{array}{l}\text { Mutations in genes that encode } \\
\text { proteins involved in glomerular } \\
\text { filtration barrier structure and function }\end{array}$ & $\begin{array}{l}\text { Cannot be established, despite } \\
\text { comprehensive evaluation }\end{array}$ \\
\hline
\end{tabular}

sparsentan on FSGS were assessed in the DUET study, which randomly assigned 109 patients with biopsy-proven FSGS or with a disease-causing mutation associated with FSGS and urine protein-tocreatinine ratio (UPCR) $>1 \mathrm{~g} / \mathrm{g}$ to receive either sparsentan or the angiotensin II type 1 receptor antagonist irbesartan ${ }^{70}$. Patients were $8-75$ years of age and average BMI was $28.5 \mathrm{~kg} / \mathrm{m}^{2}$ indicating the recruitment of obese patients. Mean UPCR was low $(3.40 \mathrm{~g} / \mathrm{g})$ and serum albumin level was high $(35 \mathrm{~g} / \mathrm{l}(3.5 \mathrm{~g} / \mathrm{dl})) .34$ patients were also on immunosuppressive therapy. After 8 weeks of treatment, sparsentan-treated patients had achieved greater reductions in UPCR and blood pressure than irbesartan-treated patients, and a higher proportion of sparsentan-treated patients achieved partial remission. The beneficial effect of sparsentan on proteinuria reduction was most prominent when baseline proteinuria was low. Specifically, patients with a UPCR $<3.5 \mathrm{~g} / \mathrm{g}$ achieved a mean decrease in proteinuria of $10.8 \%$ with irbesartan versus $46.0 \%$ with sparsentan, whereas patients with UPCR $\geq 3.5 \mathrm{~g} / \mathrm{g}$ achieved a mean decrease in proteinuria of $29.3 \%$ with irbesartan and $39.7 \%$ with sparsentan. The available evidence suggests that the DUET study population consisted of a heterogeneous group of patients with ppfFSGS, maladaptive FSGS and genetic FSGS. Indirect evidence suggests that the main benefit of sparsentan was seen in the non-ppfFSGS group and that the larger blood pressure reduction observed with sparsentan may have been primarily responsible for the observed effects. Failure to stratify the results according to FSGS subtype probably resulted in a missed opportunity to separate the haemodynamic from the presumed 'podocyte protective' effects of sparsentan. A follow-up phase III trial is currently ongoing (DUPLEX, NCT03493685) and it is intended to recruit 300 patients with FSGS ${ }^{71}$. The eligibility criteria are the same as for the DUET study.

Targeting genetic mutations. A fourth category of agents (category IV) are those developed to correct or ameliorate the injurious effects of a genetic mutation on podocyte or GBM structure or function. Such agents would potentially inhibit gainof-function mutations or replace defective genes and would by definition only be effective in FSGS that results from a genetic cause or predisposition. These approaches include gene silencing strategies such as antisense oligonucleotides and small interfering RNAs, involve CRISPR-Cas9 
technology or the transplantation of stem cells.

The G1 and G2 variants that are responsible for APOL1 nephropathy are unique in that they cause damage to the kidneys, despite the fact that the APOL1 gene does not seem to be essential for kidney development or physiological function ${ }^{30}$. Thus, the abnormal gene can probably be disabled without too much fear of untoward consequences (except perhaps an increased vulnerability to trypanosomiasis). Treatment of APOL1-transgenic mice with an APOL1-targeted antisense oligonucleotide inhibited kidney and liver expression of APOL1 and reduced proteinuria ${ }^{72}$, providing proof-of-concept that antisense oligonucleotides can effectively silence the abnormal gene. An ongoing clinical trial is aimed at evaluating the efficacy, safety and pharmacokinetics of an oral APOL1 inhibitor, VX-147, in patients with an FSGS lesion and documented APOL1 G1/G1, G2/G2 or G1/G2 genotype (NCT04340362).

\section{Protecting podocytes and promoting} regeneration. Agents that are intended to non-specifically protect injured podocytes or promote podocyte regeneration (category V) are expected to have beneficial effects in all forms of FSGS.

Several lines of experimental evidence support the notion that glucocorticoids and CNIs exert direct, protective effects on podocytes ${ }^{73}$. Glucocorticoids regulate actin cytoskeleton and slit diaphragm proteins, attenuate apoptosis and restore podocyte differentiation markers after injury $^{74}$, whereas CNIs stabilize the actin cytoskeleton by protecting synaptopodin from degradation ${ }^{28}$. Although these direct effects may have a role in certain circumstances, their clinical relevance is debatable, as ppfFSGS can rapidly recur post-transplantation - a scenario in which the standard rejection prophylaxis regimen includes glucocorticoids and CNIs - and because these drugs are ineffective in maladaptive forms of FSGS. Off-target effects on podocytes have also been described for rituximab. Part of the efficacy of rituximab in preventing post-transplantation recurrence of ppfFSGS has been attributed to restoration of the expression of sphingomyelin phosphodiesterase acid-like 3b (SMPDL-3b), which is required for correct cytoskeleton function ${ }^{73}$.

ACTH stimulates the secretion of glucocorticoids from the adrenal cortex, but is also an endogenous agonist of the melanocortin hormone system. Melanocortin receptors are abundantly expressed in the kidney, including in podocytes; stimulation of the melanocortin-1 receptor (MC1R) in podocytes stabilizes the actin cytoskeleton ${ }^{75}$. Protective effects of melanocortin agonism independent of MC1R stimulation have also been demonstrated, including prevention of apoptosis, actin cytoskeleton derangement and podocyte hypermotility, in an animal model of FSGS ${ }^{76}$. ACTH therefore has potential to reduce proteinuria through combined systemic immunomodulation and direct protection of podocytes. In an uncontrolled study of patients with 'biopsy-proven idiopathic FSGS' who were resistant to glucocorticoids (and in some instances, to other immunosuppressive agents) ACTH induced partial or complete remission in $29 \%$ of patients (5 partial remissions and 2 complete remissions, in a total of 24 patients) $)^{77}$. Nephrotic syndrome was an inclusion criterium, but was present in only 12 of 24 patients ( 10 patients had UPCR $<3.5 \mathrm{~g} / \mathrm{g}$ and 6 patients had normal serum albumin) and no EM data were provided. As all patients were resistant to glucocorticoids, any observed effect of ACTH would be expected to be related to melanocortin agonism. However, the cohort consisted of a heterogeneous group of patients, many of whom probably had secondary FSGS. Delineating which patients may benefit from melanocortin agonism is therefore not currently possible.

Abatacept is a cytotoxic

T-lymphocyte-associated protein 4 (CTLA-4)-immunoglobulin fusion molecule that targets CD80 (B7-1) on antigen-presenting cells to disrupt T-cell activation. Experimental studies suggest that upregulation of CD80 on podocytes might have a role in the pathogenesis of nephrotic syndrome, independent of T-cell activation ${ }^{78}$. Abatacept might thus represent an attractive treatment option in ppfFSGS, through its simultaneous effects on the adaptive immune response and cellular pathways of podocyte damage. However, a number of studies have been unable to locate B7-1 in podocytes or reproduce the podocyte response to abatacept, questioning the experimental data supporting this hypothesis ${ }^{78}$. A phase II RCT of abatacept in patients with therapy-resistant FSGS and MCD has now been completed, but the study findings have not yet been reported (NCT02592798).

Activation of the small GTPase RAC1 induces translocation of transient receptor potential canonical-5 (TRPC5) ion channels to the podocyte cell membrane, where they are activated by receptors such as the angiotensin II type 1 receptor ${ }^{79}$. This activation further stimulates RAC1, and sets in motion cytoskeletal changes that lead to FPE, podocyte loss and proteinuria ${ }^{80}$. Inhibition of TRPC5 reduced proteinuria in animal models of FSGS ${ }^{79,81}$. A novel TRPC5 channel inhibitor has recently completed phase I evaluation in healthy volunteers (NCT03970122). A phase IIa study (NCT04387448) is recruiting patients with diabetic nephropathy, FSGS and treatment-resistant minimal change disease - a heterogeneous mix of diseases that have very different clinical phenotypes and risk of progression, therefore requiring different surrogate end points for appropriate evaluation of the inhibitor that are not accounted for in the study design.

The regenerative potential of mesenchymal stem cells has also been demonstrated in animal models of FSGS $^{82,83}$ and it is tempting to speculate that mesenchymal stem cells may help to replenish the pool of podocytes during stress or damage. In a patient with recurrent FSGS after kidney transplantation not responsive to conventional therapy, human allogeneic bone marrow mesenchymal stem cell infusions improved proteinuria to the point that plasmapheresis could be discontinued $^{84}$. A single group intervention trial of stem cell therapy in patients with FSGS (NCT02693366) has recently been completed.

\section{Targeting inflammation and fibrosis.} Agents that are intended to suppress the inflammatory, pro-fibrotic component of the FSGS lesion (category VI) are expected to be most beneficial in the inflammatory and scarring phase of FSGS and thus be advantageous in all forms of FSGS.

The recruitment of $\mathrm{C}-\mathrm{C}$ chemokine receptor type 2 (CCR2)-expressing macrophages by $\mathrm{C}-\mathrm{C}$ motif chemokine 2 (also known as MCP-1) produced by tubule cells might contribute to glomerular and interstitial scarring in FSGS. Podocytes also express CCR2 and respond to MCP-1 with increased cellular motility, rearrangement of the actin cytoskeleton, and increased permeability to albumin ${ }^{85}$. Deficiency ${ }^{86}$ or inhibition ${ }^{87}$ of CCR2 has shown benefits in animal models of FSGS, and several trials are currently underway to evaluate the effects of CCR2 inhibitors in patients with FSGS (NCT03649152, NCT03536754, NCT03703908).

Nuclear factor erythroid 2-related factor 2 (Nrf2) is a transcription factor that regulates the expression of several hundreds of genes 
involved in defence against oxidative stress and inflammation through mechanisms that include suppression of nuclear factor- $\kappa \mathrm{B}(\mathrm{NF}-\kappa \mathrm{B})$ transcription $^{88}$. These broad cytoprotective mechanisms suggest that activators of Nrf2 may be beneficial in any condition in which oxidative stress and inflammation are part of the underlying pathophysiology. A phase II trial of the potent Nrf2 activator bardoxolone in several nephropathies, including FSGS (defined as 'biopsy-confirmed FSGS not due to known secondary causes') (NCT03366337) was completed in January 2019, but to our knowledge the results have not been published. A separate phase II RCT of CXA-10 (10-nitro-octadec-9-enoic acid) - a nitro fatty acid that also acts as a Nrf2 activator - in primary FSGS was completed in July 2020 (NCT03422510). Again, the findings have not yet been reported.

The Slit2-Roundabout (Robo) signalling pathway exerts anti-inflammatory effects by regulating leukocyte and fibroblast cytoskeletal organization, inhibiting leukocyte recruitment and inhibiting TGF- $\beta$-induced collagen synthesis by fibroblasts ${ }^{89,90}$. A phase II trial is currently underway to assess the effects of a Slit-2 antagonist - PF-06730512 - on FSGS in patients receiving 1-3 classes of immunosuppressants and UPCR $>1.5 \mathrm{~g} / \mathrm{g}$ (NCT03448692).

Losmapimod is a p38 mitogen-activated protein kinase (MAPK) inhibitor that blocks the production of inflammatory cytokines and TGF- $\beta$-induced fibrosis. In animal models of FSGS, inhibition of p38 MAPK prevents podocyte injury, proteinuria and glomerulosclerosis ${ }^{91}$. However, losmapimod failed to reduce proteinuria in a single-arm phase II trial conducted in adults with FSGS ${ }^{22}$. We concur with the authors' statement that 'study population heterogeneity may have contributed to the negative findings', which highlights the need to properly phenotype patients before recruitment.

Expression of TNF pathway genes is increased in a subset of patients with FSGS $^{93}$. In a phase I study of patients with therapy-resistant FSGS, adalimumab - a monoclonal antibody to TNF - reduced proteinuria by $\geq 50 \%$ in 4 of 10 patients after 16 weeks of treatment ${ }^{94}$. However, in a subsequent phase II trial, none of the 7 patients achieved that goal after 26 weeks of treatment ${ }^{60}$. A phase II trial of adalimumab that is aimed at recruiting 8 patients with FSGS or therapy-resistant minimal change disease is currently underway (NCT04009668).
A path forward for future trial design In our view, future treatment strategies for patients with an FSGS lesion should combine a compound that targets the cause of the lesion (those in categories I-IV) with a compound that non-specifically protects or regenerates podocytes (category V) or prevents downstream damage (category VI). Clinical trials of new therapeutic agents should add the study drug to standard pharmacological therapy (single or dual RAS blockers) and non-pharmacological approaches (dietary salt restriction), titrated to maximize proteinuria reduction and ensure blood pressure control (systolic blood pressure $\leq 120 \mathrm{mmHg})^{10}$.

Trials should only recruit patients with the FSGS subtype that is expected to derive benefit from the agent under study. If patients with different FSGS subtypes are eligible, patients should be stratified at randomization to ensure that the pathophysiological subtypes of FSGS are appropriately balanced between the active and comparator groups. Proper patient characterization at entry requires serum albumin measurements with specification of the biochemical assay, at least two measurements of proteinuria to account for technical and collection errors, EM with evaluation of FPE, and genetic analysis using the most recent FSGS gene panels or whole exon sequencing. Measurement of urinary protein excretion using a $24-\mathrm{h}$ urine collection is preferred to UPCR on a random 'spot' urine sample, but a reasonable compromise is measurement of UPCR on a spot sample of an 'intended' 24-h urine collection ${ }^{10,95,96}$. Only patients with eGFR $>30 \mathrm{ml} / \mathrm{min} / 1.73 \mathrm{~m}^{2}$ should be recruited, to exclude those with irreversible kidney damage that will likely progress, despite appropriate therapy. Proteinuria, medication use and eGFR should be stable for 3 months prior to trial entry. The delay between the kidney biopsy and enrolment should be minimal and not greater than 1 year.

We propose that trial participants should be stratified into categories of FSGS based on their clinicopathological characteristics (TABLE 2). As described earlier, no 'gold-standard' biomarker yet exists to reliably identify different subtypes of FSGS, for instance, ppfFSGS from other forms of FSGS. Until such biomarkers are available, we propose that a combination of clinical, laboratory and morphological features can be used to stratify patients. The heterogeneous population of glucocorticoid-resistant FSGS may be particularly challenging to stratify. We propose defining glucocorticoid-resistant
ppfFSGS as FSGS with the following features: the presence of diffuse FPE on EM; persistence of nephrotic syndrome with $<20 \%$ reduction of proteinuria from baseline after 8 weeks or $<50 \%$ reduction of proteinuria from baseline after 16 weeks, despite treatment with prednisolone at a dose of $1 \mathrm{mg} / \mathrm{kg}$; an absence of a family history of proteinuric kidney disease and no detection of a pathogenic variant with advanced genetic testing.

Despite the existence of some controversy, decline in proteinuria remains an accepted surrogate marker of the slowing of progression to kidney failure in clinical trials of proteinuric kidney disease $\mathrm{e}^{97,98}$. In patients with FSGS, the approach to proteinuria reduction should depend on the subtype of FSGS under study, to account for the different disease courses and baseline levels of proteinuria. For patients with ppfFSGS, a response can be defined as complete remission if proteinuria is $\leq 0.3 \mathrm{~g} / 24 \mathrm{~h}$, partial remission if proteinuria is $>0.3$ but $<3.5 \mathrm{~g} / 24 \mathrm{~h}$ and no remission if proteinuria remains $\geq 3.5 \mathrm{~g} / 24 \mathrm{~h}$ within 16 weeks, provided that eGFR remains relatively stable. It can be misleading to use the percentage in proteinuria decline from baseline to define response to therapy, as the absolute level of baseline proteinuria can vary greatly. For patients with maladaptive and genetic forms of FSGS, a $30-40 \%$ reduction in proteinuria ${ }^{99,100}$ and preservation of eGFR ${ }^{101,102}$ seem appropriate surrogate markers and therapeutic targets. Trials should have follow-up times of at least 2-3 years. A repeat biopsy at the end of the trial does not seem to be useful, because the pathological changes (such as sclerotic lesions and FPE) are not validated surrogate markers and the response to therapy can be adequately assessed by the changes in proteinuria and kidney function.

Conducting sufficiently powered RCTs in FSGS is a challenge. This challenge is particularly great for ppfFSGS, because, contrary to common belief, this is a rare disease $\mathrm{e}^{15}$. Open label studies and shared control groups could help patient recruitment, provided the stringent criteria of study design outlined in the present paper are applied. Propensity score matching ${ }^{103}$ and cluster randomized trials ${ }^{104}$ are alternative means of making it easier to conduct trials in FSGS.

\section{Conclusions}

A correct differential diagnosis between ppfFSGS, secondary (maladaptive, viral or toxic) and genetic FSGS in adults requires a clinico-pathological approach. Future 
work should aim to identify biomarkers that will more precisely reflect the underlying pathophysiological processes, but until such biomarkers are available, stratification of patients based on clinico-pathological criteria is essential, not only to facilitate sound treatment decisions for individual patients but also to facilitate the rational design of therapeutic trials. We propose that the enrolment of heterogeneous patient populations with differing underlying causes of disease has contributed to the failure of several clinical trials. The misclassification of patients in therapeutic trials invalidates conclusions from these trials and clinicians should be aware of this. We propose a model for future trial design where identification of the target population follows categorization of the compound under study and target patient recruitment is organized subsequent to a comprehensive clinicopathological assessment of the enrolled individuals.

An S. De Vriese 1,2 , Jack F. Wetzels (iD) 3 ,

Richard J. Glassock ${ }^{4}$, Sanjeev Sethi (iD

and Fernando C. Fervenza (iD) ${ }^{6 凶}$

'Division of Nephrology and Infectious Diseases, AZ Sint-Jan Brugge, Brugge, Belgium.

${ }^{2}$ Department of Internal Medicine, Ghent University, Ghent, Belgium

${ }^{3}$ Department of Nephrology, Radboud University Medical Center, Radboud Institute for Health Sciences, Nijmegen, The Netherlands.

${ }^{4}$ Department of Medicine, Geffen School of Medicine at UCLA, Los Angeles, CA, USA.

${ }^{5}$ Department of Laboratory Medicine and Pathology, Mayo Clinic, Rochester, MN, USA.

${ }^{6}$ Division of Nephrology and Hypertension,

Mayo Clinic, Rochester, MN, USA.

凶e-mail: fervenza.fernando@mayo.edu

https://doi.org/10.1038/s41581-021-00427-1

Published online 20 May 2021

1. D’Agati, V. D., Fogo, A. B., Bruijn, J. A. \& Jennette, J. C. Pathologic classification of focal segmental glomerulosclerosis: a working proposal. Am. J. Kidney Dis. 43, 368-382 (2004).

2. Velosa, J. A., Donadio, J. V. Jr \& Holley, K. E. Focal sclerosing glomerulonephropathy: a clinicopathologic study. Mayo Clin. Proc. 50, 121-133 (1975).

3. Fogo, A. B. Causes and pathogenesis of focal segmental glomerulosclerosis. Nat. Rev. Nephrol. 11, 76-87 (2015)

4. De Vriese, A. S., Sethi, S., Nath, K. A., Glassock, R. J. \& Fervenza, F. C. Differentiating primary, genetic and secondary FSGS in adults: a clinicopathologic approach. J. Am. Soc. Nephrol. 29, 759-774 (2018).

5. Kopp, J. B. et al. Podocytopathies. Nat. Rev. Dis. Prim. 13, 68 (2020).

6. Candelier, J. J. \& Lorenzo, H. K. Idiopathic nephrotic syndrome and serum permeability factors: a molecular jigsaw puzzle. Cell Tissue Res. 379, 231-243 (2020).

Savin, V. J. et al. Multiple targets for novel therapy of FSGS associated with circulating permeability factor Biomed. Res. Int. 2017, 6232616 (2017).

8. den Braanker, D. J. W. et al. Novel in vitro assays to detect circulating permeability factor(s) in idiopathic focal segmental glomerulosclerosis. Nephrol. Dial. Transplant. 6, gfaa211 (2020).

9. $\mathrm{Li}, \mathrm{M}$. et al. Assessment of increased glomerular permeability associated with recurrent focal segmental glomerulosclerosis using an in vitro model of the glomerular filtration barrier. J. Nephrol. 33, 747-755 (2020).
10. Kidney Disease: Improving Global Outcomes (KDIGO) Glomerular Diseases Work Group. KDIGO 2021 Clinical practice guideline on glomerular diseases. Kidney Int. 99 (3S), S1-87 (2021).

11. Korbet, S. M. Treatment of primary FSCS in adults. J. Am. Soc. Nephrol. 23, 1769-1776 (2012).

12. Praga, M. et al. Nephrotic proteinuria without hypoalbuminemia: clinical characteristics and response to angiotensin-converting enzyme inhibition. Am. J. Kidney Dis. 17, 330-338 (1991).

13. Maas, R. J., Deegens, J. K., van den Brand, J. A. Cornelissen, E. A. \& Wetzels, J. F. A retrospective study of focal segmental glomerulosclerosis: clinical criteria can identify patients at high risk for recurrent disease after first renal transplantation. BMC Nephrol. 14, 47 (2013).

14. Maas, R. J., Deegens, J. K., Smeets, B., Moeller, M. J. $\&$ Wetzels, J. F. Minimal change disease and idiopathic FSCS: manifestations of the same disease. Nat. Rev. Nephrol. 12, 768-776 (2016).

15. Hommos, M. S. et al. The incidence of primary vs secondary focal segmental glomerulosclerosis: a clinicopathologic study. Mayo Clin. Proc. 92, 1772-1781 (2017)

16. Kudose, S. et al. Kidney biopsy findings in patients with COVID-19. J. Am. Soc. Nephrol. 31, 1959-1968 (2020).

17. Lepori, N., Zand, L., Sethi, S., Fernandez-Juarez, G. $\&$ Fervenza, F. C. Clinical and pathological phenotype of genetic causes of focal segmental glomerulosclerosis in adults. Clin. Kidney J. 11, 179-190 (2018).

18. Gast, C. et al. Collagen (COL4A) mutations are the most frequent mutations underlying adult focal segmental glomerulosclerosis. Nephrol. Dial. Transpl. 31, 961-970 (2016).

19. Sol, M. et al. Glomerular endothelial cells as instigators of glomerular sclerotic diseases. Front. Pharmacol. 11, 573557 (2020).

20. Landini, S. et al. Reverse phenotyping after whole-exome sequencing in steroid-resistant nephrotic syndrome. Clin. J. Am. Soc. Nephrol. 15, 89-100 (2020).

21. Miao, J. et al. Positive identification of genetic causes of FSCS increases with proper patient selection Mayo Clin Proc. In press (2021).

22. Wang, M. et al. Contributions of rare gene variants to familial and sporadic FSGS. J. Am. Soc. Nephrol. 30 1625-1640 (2019).

23. Daina, E. et al. A multidrug, antiproteinuric approach to Alport syndrome: a ten-year cohort study. Nephron 130, 13-20 (2015).

24. Gee, H. Y. et al. Mutations in EMP2 cause childhoodonset nephrotic syndrome. Am. J. Hum. Genet. 94 884-890 (2014).

25. Ashraf, S. et al. Mutations in six nephrosis genes delineate a pathogenic pathway amenable to treatment. Nat. Commun 9, 1960 (2018).

26. Büscher, A. K. et al. German Pediatric Nephrology Association (GPN). Rapid response to cyclosporin A and favorable renal outcome in nongenetic versus genetic steroid-resistant nephrotic syndrome. Clin. J. Am. Soc. Nephrol. 11, 245-253 (2016).

27. Malakasioti, G., Iancu, D. \& Tullus, K. Calcineurin inhibitors in nephrotic syndrome secondary to podocyte gene mutations: a systematic review. Pediatr. Nephrol. https://doi.org/10.1007/s00467020-04695-0 (2020)

28. Faul, C. et al. The actin cytoskeleton of kidney podocytes is a direct target of the antiproteinuric effect of cyclosporine A. Nat. Med. 14, 931-938 (2008).

29. Patrakka, J et al. Recurrence of nephrotic syndrome in kidney grafts of patients with congenital nephrotic syndrome of the Finnish type: role of nephrin. Transplantation 73, 394-403 (2002).

30. Friedman, D. J. \& Pollak, M. R. APOL1 nephropathy: from genetics to clinical applications. Clin. J. Am. Soc. Nephrol. 2, 15161219 (2020).

31. Korkmaz, E. et al. ADCK4-associated glomerulopathy causes adolescence-onset FSGS. J. Am. Soc. Nephrol. 27, 63-68 (2016)

32. Tin, A. et al. Association between mitochondrial DNA copy number in peripheral blood and incident CKD in the atherosclerosis risk in communities study. J. Am. Soc. Nephrol. 27, 2467-2473 (2016).

33. Kriz, W. The inability of podocytes to proliferate: cause, consequences, and origin. Anat. Rec. 303 2588-2596 (2020).

34. Smeets, B. et al. The parietal epithelial cell: a key player in the pathogenesis of focal segmental glomerulosclerosis in Thy-1.1 transgenic mice. J. Am. Soc. Nephrol. 15, 928-939 (2004).
35. Moeller, M. J. \& Tharaux, P. L. Cellular regeneration of podocytes from parietal cells: the debate is still open. Kidney Int. 96, 542-544 (2019).

36. Lasagni, L. et al. Podocyte regeneration driven by renal progenitors determines glomerular disease remission and can be pharmacologically enhanced. Stem Cell Rep. 5, 248-263 (2015).

37. Mondini, A., Messa, P. \& Rastaldi, M. P. The sclerosing glomerulus in mice and man: novel insights. Curr. Opin. Nephrol. Hypertens. 23, 239-244 (2014).

38. Kriz, W., Shirato, I., Nagata, M., LeHir, M. \& Lemley, K. V. The podocyte's response to stress: the enigma of foot process effacement. Am. J. Physiol. Renal Physiol. 304, F333-F347 (2013).

39. Ichimura, K. et al. Morphological processes of foot process effacement in puromycin aminonucleoside nephrosis revealed by FIB/SEM tomography. J. Am. Soc. Nephrol. 30, 96-108 (2019).

40. Kriz, W. \& Lemley, K. V. Mechanical challenges to the glomerular filtration barrier: adaptations and pathway to sclerosis. Pediatr. Nephrol. 32, 405-417 (2017).

41. Kriz, W. \& Lemley, K. V. Potential relevance of shear stress for slit diaphragm and podocyte function. Kidney Int. 91, 1283-1286 (2017)

42. Deegens, J. K. et al. Podocyte foot process effacement as a diagnostic tool in focal segmental glomerulosclerosis. Kidney Int. 74, 1568-1576 (2008).

43. Praga, M. et al. Absence of hypoalbuminemia despite massive proteinuria in focal segmental glomerulosclerosis secondary to hyperfiltration. Am. J. Kidney Dis. 33, 52-58 (1999).

44. Sethi, S., Glassock, R. J. \& Fervenza, F. C. Focal segmental glomerulosclerosis: towards a bette understanding for the practicing nephrologist. Nephrol. Dial. Transpl. 30, 375-384 (2015).

45. Sethi, S., Zand, L., Nasr, S. H., Glassock, R. J. \& Fervenza, F. C. Focal and segmental glomerulosclerosis: clinical and kidney biopsy correlations. Clin. Kidney J. 7, 531-537 (2014).

46. Kambham, N., Markowitz, G. S., Valeri, A. M., Lin, J. \& D’Agati, V. D. Obesity-related glomerulopathy: an emerging epidemic. Kidney Int. 59, 1498-1509 (2001).

47. Zhong, Y. et al. The evolution of morphological variants of focal segmental glomerulosclerosis: a repeat biopsy-based observation. Nephrol. Dial. Transpl. 31, 87-95 (2016).

48. Taneda, S. et al. Podocyte and endothelial injury in focal segmental glomerulosclerosis: an ultrastructura analysis. Virchows Arch. 467, 449-458 (2015).

49. Siegerist, F., Endlich, K. \& Endlich, N. Novel microscopic techniques for podocyte research. Front. Endocrinol. 9, 379 (2018).

50. Artelt, N. et al. Comparative analysis of podocyte foot process morphology in three species by 3D superresolution microscopy. Front. Med. 5, 292 (2018).

51. Glassock, R. J., Fervenza, F. C., Hebert, L. \& Cameron, J. S. Nephrotic syndrome redux. Nephrol. Dial. Transpl. 30, 12-17 (2015).

52. van de Logt, A. E. et al. The bias between different albumin assays may affect clinical decision-making. Kidney Int. 95, 1514-1517 (2019).

53. Maas, R. J., Deegens, J. K. \& Wetzels, J. F Permeability factors in idiopathic nephrotic syndrome historical perspectives and lessons for the future. Nephrol. Dial. Transpl. 29, 2207-2216 (2014).

54. Koehler, S et al. Proteome analysis of isolated podocytes reveals stress responses in glomerular sclerosis. J. Am. Soc. Nephrol. 31, 544-559 (2020).

55. Oliverio, A. L., Bellomo, T. \& Mariani, L. H. Evolving clinical applications of tissue transcriptomics in kidney disease. Front. Pediatr. 7, 306 (2019).

56. Kashgary, A. et al. The role of plasma exchange in treating post-transplant focal segmental glomerulosclerosis: a systematic review and meta-analysis of 77 case-reports and case-series. BMC Nephrol. 17, 104 (2016).

57. Dantal, J. et al. Antihuman immunoglobulin affinity immunoadsorption strongly decreases proteinuria in patients with relapsing nephrotic syndrome. J. Am. Soc. Nephrol. 9, 1709-1715 (1998).

58. Kronbichler, A. et al. Immunoadsorption in nephrotic syndrome: where are we now and where are we going from here? Atheroscler. Suppl. 40, 55-60 (2019).

59. Savin, V. J., McCarthy, E. T., Sharma, R., Charba, D. \& Sharma, M. Galactose binds to focal segmental glomerulosclerosis permeability factor and inhibits its activity. Transl. Res. 151, 288-292 (2008).

60. Trachtman, H. et al. Efficacy of galactose and adalimumab in patients with resistant focal segmental 
glomerulosclerosis: report of the font clinical trial group. BMC Nephrol. 16, 111 (2015).

61. Kairaitis, L. et al. Blockade of CD40-CD40 ligand protects against renal injury in chronic proteinuric renal disease. Kidney Int. 64, 1265-1272 (2003).

62. Delville, M. et al. A circulating antibody panel for pretransplant prediction of FSGS recurrence after kidney transplantation. Sci. Transl. Med. 6, 256 ra136 (2014).

63. Gipson, D. S. et al. Clinical trial of focal segmental glomerulosclerosis in children and young adults. Kidney Int. 80, 868-878 (2011).

64. Troost, J. P. et al. Proteinuria reduction and kidney survival in focal segmental glomerulosclerosis. Am. J. Kidney Dis. 77, 216-225 (2020).

65. Fernandez-Fresnedo, G. et al. Rituximab treatment of adult patients with steroid resistant focal segmental glomerulosclerosis. Clin. J. Am. Soc. Nephrol. 4 1317-1323 (2009)

66. Fervenza, F. C. \& Sethi, S. Frequent-relapsing, steroid-dependent minimal change disease: is rituximab the answer? Nephrol. Dial. Transpl. 29, 722-727 (2014)

67. Alhasan, K. A., Alherbish, A., Osman, A., Kari, J. A. \& Almojalli, H. Successful treatment of recurrent focal segmental glomerulosclerosis after transplantation in children: a single-center experience. Transpl. Proc. $\mathbf{5 1}$ 517-521 (2019).

68. Nishiyama, A. \& Kobori, H. Independent regulation of renin-angiotensin-aldosterone system in the kidney. Clin. Exp. Nephrol. 22, 1231-1239 (2018).

69. Kohan, D. E. \& Barton, M. Endothelin and endothelin antagonists in chronic kidney disease. Kidney Int. 86, 896-904 (2014)

70. Trachtman, $\mathrm{H}$. et al. DUET: a phase 2 study evaluating the efficacy and safety of Sparsentan in patients with FSGS. J. Am. Soc. Nephrol. 29, 2745-2754 (2018).

71. Komers, R. et al. Study design of the phase 3 Sparsentan versus Irbesartan (DUPLEX) study in patients with focal segmental glomerulosclerosis. Kidney Int. Rep. 5, 494-502 (2020).

72. Aghajan, M. et al. Antisense oligonucleotide treatment ameliorates IFN- $\gamma$-induced proteinuria in APOL1transgenic mice. JCl Insight 4, e 126124 (2019).

73. Yoo, T. H. \& Fornoni, A. Nonimmunologic targets of immunosuppressive agents in podocytes. Kidney Res. Clin. Pract. 34, 69-75 (2015).

74. Mallipattu, S. K. et al. Kruppel-like Factor 15 mediates glucocorticoid-induced restoration of podocyte differentiation markers. J. Am. Soc. Nephrol. 28 166-184 (2017).

75. Bergwall, L. et al. Amplification of the melanocortin-1 receptor in nephrotic syndrome identifies a target for podocyte cytoskeleton stabilization. Sci. Rep. $\mathbf{8}$, 15731 (2018).

76. Qiao, Y. et al. Melanocortin therapy ameliorates podocytopathy and proteinuria in experimental focal segmental glomerulosclerosis involving a podocyte specific non-MC1R-mediated melanocortinergic signaling. Clin. Sci. 134, 695-710 (2020).

77. Hogan, J. et al. Treatment of idiopathic FSGS with adrenocorticotropic hormone gel. Clin. J. Am. Soc. Nephrol. 8, 2072-2081 (2013).
78. Novelli, R., Benigni, A. \& Remuzzi, G. The role of B7-1 in proteinuria of glomerular origin. Nat. Rev. Nephrol. 14, 589-596 (2018)

79. Zhou, Y. et al. A small-molecule inhibitor of TRPC5 ion channels suppresses progressive kidney disease in animal models. Science 358, 1332-1336 (2017).

80. van der Wijst, J. \& Bindels, R. J. M. Renal physiology: TRPC5 inhibition to treat progressive kidney disease. Nat. Rev. Nephrol. 14, 145-146 (2018).

81. Yu, M. et al. Discovery of a potent and selective TRPC5 Inhibitor, efficacious in a focal segmental glomerulosclerosis model. ACS Med. Chem. Lett. 10, 1579-1585 (2019).

82. Yang, R. C. et al. Bone marrow mesenchymal stem cells attenuate the progression of focal segmental glomerulosclerosis in rat models. BMC Nephrol. 19 335 (2018).

83. Ornellas, F. M. et al. Mesenchymal stromal cells induce podocyte protection in the puromycin injury model. Sci. Rep. 9, 19604 (2019).

84. Belingheri, M. et al. Allogeneic mesenchymal stem cell infusion for the stabilization of focal segmental glomerulosclerosis. Biologicals 41, 439-445 (2013).

85. Lee, E. Y. et al. The monocyte chemoattractant protein-1/CCR2 loop, inducible by TGF-beta, increases podocyte motility and albumin permeability. Am. J. Physiol. Renal Physiol. 297, F85-F94 (2009).

86. Wilkening, A. et al. C-C chemokine receptor type 2 mediates glomerular injury and interstitial fibrosis in focal segmental glomerulosclerosis. Nephrol. Dial. Transpl. 35, 227-239 (2020).

87. Miao, Z. et al. CCR2 antagonism leads to marked reduction in proteinuria and glomerular injury in murine models of focal segmental glomerulosclerosis (FSGS). PLoS One 13, e0192405 (2018).

88. Ito, M., Tanaka, T. \& Nangaku, M. Nuclear factor erythroid 2-related factor 2 as a treatment target of kidney diseases. Curr. Opin. Nephrol. Hypertens. 29, 128-135 (2020).

89. Chaturvedi, S. \& Robinson, L. A. Slit2-Robo signaling in inflammation and kidney injury. Pediatr. Nephrol. 30, 561-566 (2015)

90. Yuen, D. A. et al. Recombinant N-terminal Slit2 inhibits TGF- $\beta$-induced fibroblast activation and renal fibrosis. J. Am. Soc. Nephrol. 27, 2609-2615 (2016)

91. Koshikawa, M. et al. Role of p38 mitogen-activated protein kinase activation in podocyte injury and proteinuria in experimental nephrotic syndrome. J. Am. Soc. Nephrol. 16, 2690-2701 (2005).

92. Gipson, D. S. et al. Assessing the impact of Losmapimod on proteinuria in idiopathic focal segmental glomerulosclerosis. Kidney Int. Rep. 5, 1228-1239 (2020).

93. Chung, C. F. et al. Intrinsic tumor necrosis factor-alpha pathway is activated in a subset of patients with focal segmental glomerulosclerosis. PLoS One 14 e0216426 (2019).

94. Joy, M. S. et al. Phase 1 trial of adalimumab in focal segmental glomerulosclerosis (FSGS): II. Report of the FONT (Novel therapies for resistant FSCS) study group. Am. J. Kidney Dis. 55, 50-60 (2010).

95. Hogan, M. C. et al. The relatively poor correlation between random and 24-hour urine protein excretion in patients with biopsy-proven glomerular diseases. Kidney Int. 90, 1080-1089 (2016).

96. Kaminska, J., Dymicka-Piekarska, V., Tomaszewska, J., Matowicka-Karna, J. \& Koper-Lenkiewicz, O. M Diagnostic utility of protein to creatinine ratio ( $\mathrm{P} / \mathrm{C}$ ratio) in spot urine sample within routine clinical practice. Crit. Rev. Clin. Lab. Sci. 57, 345-364 (2020).

97. Glassock, R. J. Albuminuria: a target for clinical trials in kidney disease? Nat. Rev. Nephrol. 15, 257-258 (2019).

98. Thompson, A., Smith, K. \& Lawrence, J. Change in estimated GFR and albuminuria as End Points in Clinical Trials: a viewpoint from the FDA. Am. J. Kidney Dis. 75, 4-5 (2020).

99. Heerspink, H. J. L. et al. Chronic Kidney Disease Epidemiology Collaboration. Change in albuminuria as a surrogate endpoint for progression of kidney disease: a meta-analysis of treatment effects in randomised clinical trials. Lancet Diabetes Endocrinol. 7, 128-139 (2019).

100. Coresh, J. et al. Change in albuminuria and subsequent risk of end-stage kidney disease: an individual participant-level consortium meta-analysis of observational studies. Lancet Diabetes Endocrinol. 7, 115-127 (2019).

101. Grams, M. E et al. Evaluating glomerular filtration rate slope as a surrogate end point for ESKD in clinical trials: an individual participant meta-analysis of observational data. J. Am. Soc. Nephrol. 30, 1746-1755 (2019).

102. Greene, T. et al. Performance of GFR slope as a surrogate end point for kidney disease progression in clinical trials: a statistical simulation. J. Am. Soc. Nephrol. 30, 1756-1769 (2019).

103. Dekkers, I. A. \& van der Molen, A. J. Propensity score matching as a substitute for randomized controlled trials on acute kidney injury after contrast media administration: a systematic review. AJR Am. J. Roentgenol. 211, 822-826 (2018)

104. Nicholls, S. G. et al. Ethical issues in the design and conduct of pragmatic cluster randomized trials in hemodialysis care: an interview study with key stakeholders. Can. J. Kidney Health Dis. 7 2054358120964119 (2020).

\section{Author contributions}

A.S.D.V and F.C.F. wrote the article. All authors researched data for the article, contributed substantially to discussion of the content and reviewed and/or edited the manuscript before submission.

\section{Competing interests}

The authors declare no competing interests.

\section{Peer review information}

Nature Reviews Nephrology thanks J. Kopp and the other, anonymous, reviewer(s) for their contribution to the peer review of this work.

Publisher's note

Springer Nature remains neutral with regard to jurisdictional claims in published maps and institutional affiliations.

(C) Springer Nature Limited 2021 